\section{Braz J Med Biol Res, J une 2010, Volume 43(6) 593-599}

doi: 10.1590/S0100-879X2010007500048

\section{Sentinel node biopsy in breast cancer: results in a large series}

S.M.T. Carvalho, M. Mourão Netto, E.N.P. Lima, A.M. Pimentel, F.B. Makdissi, C.A.B.T. Osório, M.S. Maciel, H. Iyeyasu, J.B. Collins, C.M. Fontes, A.L. Perina and F.A. Soares
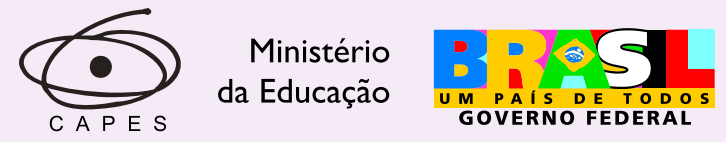

DFAPESP

Institutional Sponsors
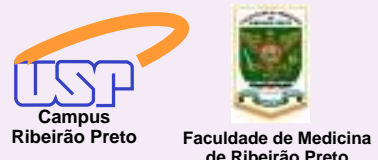

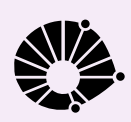

Ф SHIMADZU

GE Healthcare

Hotsite of proteomics metabolomics developped by: 


\title{
Sentinel node biopsy in breast cancer: results in a large series
}

\author{
S.M.T. Carvalho, M. Mourão Netto, E.N.P. Lima, A.M. Pimentel, F.B. Makdissi, \\ C.A.B.T. Osório, M.S. Maciel, H. Iyeyasu, J.B. Collins, C.M. Fontes, \\ A.L. Perina and F.A. Soares \\ Hospital do Câncer A.C. Camargo, São Paulo, SP, Brasil
}

\begin{abstract}
Sentinel lymph node biopsy (SLNB) is an appropriate method for the evaluation of axillary status in cases of early breast cancer. We report our experience in treating cases evaluated using SLNB. We analyzed a total of 1192 cases assessed by means of SLNB from July 1999 to December 2007. SLNB processing was successfully completed in 1154 cases with the use of blue dye or radiolabeled $99 \mathrm{mTc}$-Dextran-500, or both. Of these 1154 patients, 857 were N0(i-) (no regional lymph node metastasis, negative immunohistochemistry, IHC), 96 were $\mathrm{NO}(\mathrm{i}+$ ) (no regional lymph node metastasis histologically, positive IHC, no IHC cluster greater than $0.2 \mathrm{~mm}$ ) and 201 were N1mi (greater than $0.2 \mathrm{~mm}$, none greater than $2.0 \mathrm{~mm}$ ). Most of the tumors (70\%) were invasive ductal carcinomas and tumors were staged as T1 in 770 patients (65\%). A total of 274 patients underwent SLNB and axillary dissections up to April 2003. The inclusion criteria were tumor size equal to or less than $3 \mathrm{~cm}$ in diameter, no clinically palpable axillary lymph nodes, no neoadjuvant therapy. In 19 cases, the SLN could not be identified intraoperatively. A false-negative rate of $11 \%$ and a negative predictive value of $88.2 \%$ were obtained for the 255 assessable patients. The overall concordance between SLNB and axillary lymph node status was $92 \%$. SLNB sensitivity for nodes was $81 \%$ and specificity was $100 \%$. The higher sensitivity, specificity, accuracy, and lower false-negative rates of SLNB suggest that this method may be an appropriate alternative to total axillary dissection in early breast cancer patients.
\end{abstract}

Key words: Breast neoplasms; Sentinel lymph node; Sentinel lymph node biopsy; Completion axillary dissection

\section{Introduction}

The disease status of axillary lymph nodes is the most important prognostic factor for patients with breast cancer. The sentinel lymph node biopsy (SLNB) was introduced into clinical practice in the 1990s (1-3). It is an extremely sensitive and specific method for predicting whether malignant neoplasms such as breast cancer have metastasized to regional lymph nodes $(4,5)$. According to the American Society of Clinical Oncology (ASCO), SLNB is a safe and accurate method for identifying early breast cancer without the involvement of axillary lymph nodes (6).

The histopathological analytical methods for excised sentinel lymph nodes (SLN) have been improved in order to increase staging accuracy and reduce false-negative rates $(7,8)$. The relevant SLN pathology must include multisection staining with hematoxylin and eosin (H\&E). Immunohistochemical analysis (IHC) is also commonly used for H\&E-negative cases and may facilitate the detection of small deposits, thereby enhancing the screening of SLN sections (7). The majority of SLN cases can be classified as positive or negative based on the presence or absence of macrometastasis. The 2003 edition of the TNM classification uses $2.0 \mathrm{~mm}$ as the cutoff size that distinguishes between micro- and macrometastasis (9). The cutoff value for isolated tumor cells or so-called submicrometastasis is $0.2 \mathrm{~mm}(10)$. The significance and practical relevance of micrometastases, isolated tumor cells and the value of $\mathrm{IHC}$ also require further definition. Furthermore, the use of nomograms enhances the prediction of non-SLN status in SLN-positive patients $(11,12)$.

Certain questions persist regarding the use of this technique in breast cancer. Specifically, we need to know more about axillary recurrence cases, overall survival rates and local morbidity reduction. Answers to these questions might be addressed in an oncology institution where protocols for patient treatment are strictly followed and full records can be retrospectively analyzed. In this paper, we report our

Correspondence: S.M.T. Carvalho, Departamento de Cirurgia da Mama, Hospital do Câncer A.C. Camargo, Av. Pavão, 130, Apto. 142, 04516-010 São Paulo, SP, Brasil. E-mail: solcarvalho@uol.com.br

Received July 31, 2009. Accepted May 6, 2010. Available online May 28, 2010. Published June 11, 2010. 
experience with SLNB in patients with breast cancer treated at our hospital. We analyzed the clinical and pathological features and the treatment methods used in order to determine whether the SLNB and the type of axillary surgery may impact local recurrence.

\section{Patients and Methods}

Between July 1999 and December 2007, a total of 1192 patients were treated at the Department of Breast Surgery at the Hospital do Câncer A.C. Camargo (HC-ACC, São Paulo, SP, Brazil) and underwent the SLNB procedure. Inclusion criteria were: 1) tumor size histologically equal to or less than $3 \mathrm{~cm}$ in diameter, 2) absence of clinically palpable axillary lymph nodes, 3) no neoadjuvant therapy, 4) no pregnancy, and 5) no previous ipsilateral breast cancer or axillary surgery other than benign excision biopsy.

The clinical pathology characteristics were: 1) patient characteristics, age, hormonal status; 2) breast tumor histologic type, size, Scarff Bloom Richardson grade (SBR), lymph vascular invasion (LVI), hormonal receptors; 3) SLN characteristics, number of SLN removed, intraoperative biopsy, analytical methods for SLN, IHC, and micrometastasis size; 4) number of lymph nodes analyzed after axillary lymph node dissection (ALND) and number of metastatic non-sentinel nodes, and 5) identification procedures for SLN using blue dye and/or isotope.

Tumors were classified histologically according to the American Joint Cancer Commission staging system $(10,13)$.

SLNB was performed using an isotope alone, blue dye alone or a combination of the two agents. One day before surgery or on the morning of the procedure, the patient received a $0.1-0.2-\mathrm{mL}$ injection containing 0.2-0.4 $\mathrm{mCi}$ (74-148 MBq) ${ }^{99 m} \mathrm{Tc}-$ Dextran-500 at four peritumoral sites, or as close as possible to the biopsy site in cases of a preexisting biopsy. In multifocal breast cancer, patients were injected in the periareolar area.

After injection, the patient was instructed to perform gentle circular and axillary-directed massage at the injection site and on the whole breast during the subsequent 30 to $45 \mathrm{~min}$ in order to facilitate lymphatic drainage.

Images of the thoracic region at anterior and oblique projections, including the involved breast and axillary area, were acquired using a gamma camera with a low-energy high-resolution collimator and an energy peak at $140 \mathrm{KeV}$ at least $90 \mathrm{~min}$ after the injection.

An ink dot was marked on the skin, located immediately above the projection of the first node that became radioactive and was visible on the gamma camera monitor; this node was usually the only one detected by the gamma-camera or otherwise it exhibited the greatest activity when more than one node was detected.

Just after the induction of anesthesia, 3 to $5 \mathrm{~mL}$ of a $1 \%$ solution of patent blue V (bleu patenté V, Guerbet,
France) was subdermally injected above the tumor, followed by breast massage for $5 \mathrm{~min}$. Approximately $15 \mathrm{~min}$ after patent blue injection, axillary exploration for blue-stained or radioactive nodes was performed.

A gamma detecting probe (Navigator USSC, USA) was used first on the skin over the previously marked sentinel projection to confirm the presence of radioactivity (a hot spot). Cumulative radioactive counts were obtained for 10 $s$ and the sentinel node was defined as any node showing radioactive counts with or without blue-stain. The presence of less than $10 \%$ of the initial counts at axilla was used as a parameter to consider it as a sentinel node-free area. Dissection continued until radioactive and/or blue-stained nodes had been removed and the background count of the axilla was less than $10 \%$ that of the most radioactive lymph node ex vivo.

The excised lymph nodes were submitted to histological examination. All SLN were bisected or sectioned at 2-3 $\mathrm{mm}$ for the preparation of imprint cytology. The other half was fixed with $10 \%$ formalin buffer for 6 to $10 \mathrm{~h}$ and embedded in paraffin for postoperative investigation using H\&E staining. Lymph nodes less than $3 \mathrm{~mm}$ in diameter were embedded uncut.

Three pairs of paraffin-embedded sections, 4- $\mu \mathrm{m}$ thick, were cut at 30-50- $\mu$ m intervals. One section of each pair was stained with $\mathrm{H} \& \mathrm{E}$. If no carcinoma cells were detected, we performed IHC with a cytokeratin AE1/AE3 antibody using a standard immunoperoxidase method. This antibody is directed against low- and high-molecular weight cytokeratin filaments. Before 2004, we did not perform intraoperative imprint cytology (IIC).

The size of the metastasis was determined by the largest cohesive or contiguous sheet of tumor cells, and micrometastases were measured using an ocular micrometer. Tumor cells were identified on either H\&E-stained paraffin sections or by IHC. Individual or isolated cytokeratin-positive tumor cells were $<0.2 \mathrm{~mm}$ in diameter.

When no axillary SLN was identified by either means, an axillary LN dissection was performed. Non-sentinel lymph nodes were all examined by standard histopathology. In general, an immediate ALND was performed for all patients whose SLN tested positive when using IIC. If the SLN tested negative in intraoperative sections, but was found to contain macrometastases in the final histopathology, patients underwent delayed completion level I and II ALND. Between July 1999 and April 2003, 255 patients who underwent SLNB had their axillary lymph node dissected immediately thereafter. After 2003, if SLN tested negative according to both IIC and paraffin analysis, the patients underwent SLNB without ALND.

Between July 1999 and December 2007, 1192 patients underwent the SLNB procedure. In 38 cases (19 cases before and 19 cases after April 2003), the SLN was identified weakly or not at all, both preoperatively and intraoperatively with the blue dye and/or the probe. 
The mean age of the study population was 54.3 years (range: 22 to 92 years) and approximately $41 \%$ of the patients were premenopausal. The tumor was staged as T1 in 770 patients $(65 \%)$ and as T2 in 259 patients $(21.8 \%)$. Most of the tumors (72.2\%) were invasive ductal carcinomas, $7.9 \%$ were invasive lobular carcinomas, $6.5 \%$ were classified as different types of carcinomas (atypical medullary, medullary, adenoid cystic, tubular, mucinous, apocrine, and metaplastic) and $13 \%$ were ductal carcinomas in situ (DCIS). Fifty-one percent of the tumors were grade II of SBR and $82 \%$ had no LVI. Detailed patient and tumor characteristics are shown in Table 1.

The descriptive statistics are reported as distribution of frequencies for categorical data and central trend and dispersion measures for numerical variables. Diagnostic tests were used to determine the specificity and sensitivity of SLNB. Statistical analyses were performed using the STATA software release 7.0.

\section{Results}

The SLN procedure was successfully completed in $96.8 \%$ of the cases $(1154 / 1192)$ with the use of blue dye, radio labeled $99 \mathrm{~m}$ Tc-Dextran-500 or a combination of both. Initially, we used a combination of the two techniques, and with this approach SLN were identified by blue dye in $89 \%$ $(502 / 564)$ and by radioisotope in $95 \%$ (1092/1154) of all cases. In 62 cases, the SLN were not identified by the isotope (5\%; Table 2). This is because the scintigraphy revealed very faint or no radioactivity at the axilla and the SLN could not be identified with the gamma probe the next day.

The number of SLN identified at the time of surgery is reported in Table 2. Five hundred and seventy-four patients (49\%) had only one SLN removed, while 68 (6\%) had four or more SLN removed.

A total of 766 cases were sent for IIC analysis. The SLN were positive in $12 \%$, negative in $88 \%$, and inconclusive in $1.8 \%(N=14)$ of cases. The final histological examination revealed absence of cancer in $578(85.7 \%)$ of the 674 $(88 \%)$ cases with a negative SLN at the time of IIC, whereas micrometastases were found in 58 cases $(8.6 \%)$ and macrometastases in 38 cases $(5.6 \%)$. The IIC false-negative rate was $14.2 \%$. All positive cases were confirmed at final histological examination. Of the 14 inconclusive cases, 2 tested positive for macrometastases, 6 were positive for micrometastases, and 6 confirmed the absence of cancer cells (Table 3 ).

In $14.2 \%$ of the cases, there was just one positive SLN (164/1154). Axillary lymph nodes were the most common site of SLN localization (96\%; Table 3).

Two hundred and seventy-four patients underwent axillary dissection prior to April 2003. In 19 cases, the SLN was not identified intraoperatively. Among the 255 assessable patients, the SLNB was positive for macrometastasis in 63 cases and positive for micrometastasis in 22 cases (Table 4).
Table 1. Descriptive characteristics of 1192 patients and tumors.

\begin{tabular}{|c|c|}
\hline Variable & Frequency \\
\hline \multicolumn{2}{|l|}{ Age (years) } \\
\hline Range & $22-92$ \\
\hline Median & 53 \\
\hline Mean & 54.3 \\
\hline \multicolumn{2}{|l|}{ Hormonal status } \\
\hline Pre & $487(40.9 \%)$ \\
\hline Post & $705(59.1 \%)$ \\
\hline \multicolumn{2}{|l|}{ Histologic subtype } \\
\hline IDC & $861(72.2 \%)$ \\
\hline ILC & $94(7.9 \%)$ \\
\hline DCIS & $156(13.1 \%)$ \\
\hline $\mathrm{OHT}$ & $78(6.5 \%)$ \\
\hline LCIS & $3(0.3 \%)$ \\
\hline \multicolumn{2}{|l|}{ Tumor size } \\
\hline Tis & $159(13.0 \%)$ \\
\hline T1mic & $30(2.5 \%)$ \\
\hline $\mathrm{T} 1 \mathrm{a}$ & $63(5.3 \%)$ \\
\hline $\mathrm{T} 1 \mathrm{~b}$ & $215(18.1 \%)$ \\
\hline $\mathrm{T} 1 \mathrm{c}$ & $462(38.9 \%)$ \\
\hline T2 (up to $3.0 \mathrm{~cm}$ ) & $259(21.8 \%)$ \\
\hline Unknown & $4(0.4 \%)$ \\
\hline \multicolumn{2}{|l|}{ Histologic grade } \\
\hline 1 & $243(26.9 \%)$ \\
\hline 2 & $462(51.2 \%)$ \\
\hline 3 & $198(21.9 \%)$ \\
\hline \multicolumn{2}{|l|}{ Nuclear grade } \\
\hline 1 & $96(9.6 \%)$ \\
\hline 2 & $447(44.7 \%)$ \\
\hline 3 & $457(45.7 \%)$ \\
\hline \multicolumn{2}{|c|}{ Lymphovascular invasion } \\
\hline No & $829(81.8 \%)$ \\
\hline Yes & $184(18.2 \%)$ \\
\hline \multicolumn{2}{|l|}{ Perineural invasion } \\
\hline No & $894(88.3 \%)$ \\
\hline Yes & $118(11.7 \%)$ \\
\hline \multicolumn{2}{|l|}{ Estrogen receptor } \\
\hline No & $202(17.3 \%)$ \\
\hline Yes & $962(82.7 \%)$ \\
\hline \multicolumn{2}{|l|}{ Progesterone receptor } \\
\hline No & $348(30.0 \%)$ \\
\hline Yes & $813(70.0 \%)$ \\
\hline
\end{tabular}

IDC = invasive ductal carcinoma; ILC = invasive lobular carcinoma; $\mathrm{DCIS}=$ ductal carcinoma in situ; $\mathrm{OHT}=$ other histologic types such as tubular, colloid, medullary types; LCIS = lobular carcinoma in situ; Tis = ductal carcinoma in situ; $\mathrm{T} 1 \mathrm{mic}=$ microinvasion of $0.1 \mathrm{~cm}$ or less in greatest dimension; $\mathrm{T} 1 \mathrm{a}=$ tumor greater than $0.1 \mathrm{~cm}$ but not greater than $0.5 \mathrm{~cm}$ in dimension; T1b $=$ tumor greater than $0.5 \mathrm{~cm}$ but not greater than $1 \mathrm{~cm}$ in dimension; T1c = tumor greater than $1 \mathrm{~cm}$ but not greater than $2 \mathrm{~cm}$ in dimension; $\mathrm{T} 2$ = tumor greater than $2 \mathrm{~cm}$ but not greater than 3 $\mathrm{cm}$ in dimension. 
All 22 patients who underwent level I and II ALND showed a non-SLN that was completely tumor free. One hundred and seventy patients exhibited negative findings after SLNB; in 20 of these patients, we identified metastases in other axillary lymph nodes after the immediate completion of level I and II ALND (Table 5). Within this subset of patients, 3 cases had four or more non-SLN metastases and 17 had one to three non-SLN metastases. The tumor was a T1c in 9/20 cases and a T2 in 11/20 cases. Eighty-one percent $(206 / 255)$ of the patients who underwent ALND had no

Table 2. Distribution of clinical variables.

\begin{tabular}{lc}
\hline Variable & Frequency \\
\hline SLN identified with blue dye & \\
No & $62(11 \%)$ \\
Yes & $502(89 \%)$ \\
SLN identified with technetium & \\
No & $62(5.4 \%)$ \\
Yes & $1092(94.6 \%)$ \\
Number of lymph nodes & \\
1 & $574(49.7 \%)$ \\
2 & $376(32.6 \%)$ \\
3 & $136(11.8 \%)$ \\
4 or more & $68(5.9 \%)$ \\
Positive cytology imprint & \\
No & $674(88.0 \%)$ \\
Yes & $92(12.0 \%)$ \\
Inconclusive cytology imprint & \\
No & $752(98.2 \%)$ \\
Yes & $14(1.8 \%)$ \\
H\&E-positive SLN & \\
No & $901(78.1 \%)$ \\
Yes & $253(21.9 \%)$ \\
Immunohistochemically positive SLN & \\
Yes & $780(91.4 \%)$ \\
\hline
\end{tabular}

SLN = sentinel lymph node; H\&E = hematoxylin and eosin .

Table 4. Axillary status of patients submitted to axillary lymph node dissection after the sentinel lymph node biopsy.

\begin{tabular}{lcc}
\hline Sentinel lymph node & No. of cases & $\begin{array}{c}\text { No. of axillas with } \\
\text { positive non-SLN }\end{array}$ \\
\hline Positive micrometastases & 22 & - \\
Positive macrometastases & 63 & 29 \\
Negative & 170 & 20 \\
Total & 255 & 49 \\
\hline
\end{tabular}

other positive lymph nodes. Consequently, they would have derived no benefit from complete axillary dissection.

Twenty-six patients died of the disease and only three of these exhibited micrometastasis in SLN, while 11 presented a positive SLN. Nineteen patients are still alive with the disease at the time of this writing, and only 2 of them exhibited

Table 3. Descriptive characteristics of sentinel lymph nodes.

\begin{tabular}{lc}
\hline Variable & Frequency \\
\hline Micrometastasis size & \\
Negative & $857(72.8 \%)$ \\
$<0.2$ & $31(2.8 \%)$ \\
$0.2-1.0$ & $40(3.7 \%)$ \\
$1.1-2.0$ & $25(2.3 \%)$ \\
Macro & $201(18.4 \%)$ \\
No. of positive SLN & \\
0 & $954(82.7 \%)$ \\
1 & $164(14.2 \%)$ \\
2 & $36(3.1 \%)$ \\
Localization & \\
Axilla & $1114(96.5 \%)$ \\
Internal mammary chain & $3(0.3 \%)$ \\
Axilla and internal mammary chain & $37(3.2 \%)$ \\
Axillary dissection & \\
No & $649(54.4 \%)$ \\
Yes & $543(45.6 \%)$ \\
No. of dissected SLN & \\
1 & $574(49.7 \%)$ \\
2 & $376(32.6 \%)$ \\
3 & $136(11.8 \%)$ \\
4 or more & $68(5.9 \%)$ \\
No. of positive SLN & \\
$1-3$ & $414(76.2 \%)$ \\
$4-9$ & $83(15.3 \%)$ \\
$>9$ & $16(3.0 \%)$ \\
\hline & \\
\hline & \\
\hline &
\end{tabular}

$\mathrm{SLN}=$ sentinel lymph node.

Table 5. Sentinel lymph node evaluation and definitive status of all axillary lymph nodes in $\mathbf{2 5 5}$ patients with breast cancer.

\begin{tabular}{lcc}
\hline Sentinel lymph node & $\begin{array}{c}\text { Axillary lymph } \\
\text { nodes }\end{array}$ & $\begin{array}{c}\text { Total number of } \\
\text { patients }\end{array}$ \\
\hline Positive & Positive & $85(33 \%)$ \\
Negative & Negative & $150(59 \%)$ \\
Negative & Positive & $20(8 \%)$ \\
Total & & 255 \\
\hline
\end{tabular}

$\mathrm{SLN}=$ sentinel lymph node. 
micrometastasis in SLN. We note that a total of 11 patients who died of the disease were T2(11/26) and 10 patients were T1, while 9 individuals who are still living with the disease are T2 (9/19) and 8 are T1c (8/19; Table 6). We identified only 4 axillary recurrences across patients studied $(0.45 \%)$ and 3 of these underwent intraoperative radiotherapy. We excluded all 274 patients who underwent SLNB and axillary dissection during our initial training phase.

The false-negative rate was $11 \%$ and the negative predictive value was $88.2 \%$. The overall concordance between SLNB and axillary lymph node status was $92 \%$. The sensitivity of SLNB for nodes was $81 \%$ and the specificity was $100 \%$ (see Table 7 ).

Of our 1154 patients, 857 were NO(i-) (no regional lymph node metastasis, negative $\mathrm{IHC}$ ), 96 were $\mathrm{NO}(\mathrm{i}+)$ (no regional lymph node metastasis histologically, positive IHC, no IHC cluster greater than $0.2 \mathrm{~mm}$ ) and 201 were $\mathrm{N} 1 \mathrm{mi}$ (greater than $0.2 \mathrm{~mm}$, none greater than $2.0 \mathrm{~mm}$ ). The size of the micrometastases of the 96 patients who exhibited a positive SLN is shown in Table 3.

\section{Discussion}

This was a study with a non-randomized retrospective design. Our goal was to determine if the SLNB is an accurate procedure that can provide the physician and the patient with important information concerning staging and prognosis, and to guide postoperative treatment.

We were able to identify the SLN in $96.8 \%$ of the individuals by using blue dye and/or a radioactive tracer. Our results also show that the SLNB in early stage breast cancer is an effective and useful procedure. The method correctly predicted the state of the axilla in a high percentage of patients (92\% accuracy) and this value is sufficiently high for us to conclude that axillary dissection is probably unnecessary for patients in whom the sentinel node tests negative. We observed that the use of radiocolloid and the gamma probe improved the identification of the SLN. However, the use of intraoperative blue dye injections is relatively inexpensive and accurate and can be performed even when the colloid is not available.

Our false-negative rate was $11.7 \%$. As we gained experience, and as we reduced our false-negative rate, the use of combined methods yielded the highest identification rates and are consistent with ASCO guidelines (6). There was a notable learning curve and the surgeon's rate of SLN detection increased with experience. Although our false-negative rate is higher than that reported in the literature (the false-negative rate averaged $8.4 \%$, ranging from 0 to $29 \%$ in the literature) (6), we note that our axillary recurrence rate after a negative SLNB finding is very low $(0.45 \%)$, with a follow-up of $24-60$ months. We observed axillary recurrence after $32,21,14$, and 8 months from surgery. Our results agree with the literature findings that range from 0 to $1.4 \%$ at $14-46$ months of follow-up (14). All suspicious palpable nodes (other than SLN, in the axillar content) were submitted to intraoperative analysis.

In $54 \%$ of our cases with a positive axilla, the SLN was the only node involved with no other positive node in the axillary content. As described by Veronesi et al. (15), this finding indicates that the sentinel concept is biologically valid. If we could preselect this group of positive SLN patients, ALND may not be required. It has been noted in the literature (16) that nodal spread is a stepwise phenomenon involving the SLN - this is the first and also the most likely site of regional metastases. A nomogram has been published to predict non-SLN metastasis in patients with positive SLN using nuclear grade, LVI, multifocality, estrogen receptor status, number of positive SLN, pathological tumor size, and method of detection (IHC, serial H\&E sections, routine, and frozen) $(11,12)$. However, this model was not reliably predictive for positive non-SLN in cases with micrometastatic positive SLN.

Several studies have reported that the average number of SLN removed at the time of SLNB is three (17). In our study, almost $80 \%$ of patients had one or two SLN removed. The detection of multiple SLN may reflect a migration of dye

Table 6. Unfavorable events and deaths in the study group of 1154 patients.

\begin{tabular}{lcl}
\hline Event & $\begin{array}{c}\text { Study group } \\
(\mathrm{N})\end{array}$ & Frequency \\
\hline Events other than death $^{*}$ & 30 & $2.5 \%$ \\
Axillary recurrence $^{*}$ & 4 & $0.45 \%$ \\
Recurrence in the ipsilateral breast & 10 & $0.86 \%$ \\
Distant metastases & 16 & $1.38 \%$ \\
Death due to breast cancer & 26 & $2.25 \%$ \\
Death from other causes & 15 & $1.2 \%$ \\
Lost to follow-up & 12 & $1.03 \%$ \\
\hline
\end{tabular}

*In this group, we excluded the initial 274 patients who underwent SLNB and axillary dissection.

Table 7. Agreement between sentinel lymph node evaluation and definitive axillary lymph node status after complete axillary lymph node dissection in 255 patients.

\begin{tabular}{lc}
\hline Measure & Frequency \\
\hline False-negative & $11.7 \%$ \\
Sensitivity & $81 \%$ \\
Specificity & $100 \%$ \\
Positive predictive value & $100 \%$ \\
Negative predictive value & $88.2 \%$ \\
Overall accuracy & $92.1 \%$ \\
\hline
\end{tabular}


or isotope from the true SLN to secondary lymph nodes or simply a normal anatomic variation in which the lymphatics of a given site in the breast drain simultaneously (17).

Another important consideration concerns IIC as an intraoperative method for examining the SLN. IIC can provide clear cytological detail and lead to a rapid diagnosis, preserving the tissue for permanent section, but has disadvantages in that the number of cells examined is small and the chance of an indeterminate result is high $(18,19)$. The use of H\&E staining and IHC analysis with antibodies to cytokeratin has improved the identification of metastasis in SLN. IHC evaluation can upstage disease for approximately $10 \%$ of patients who have a negative SLN (6). Lymph node metastases have traditionally been divided into those measuring $>2 \mathrm{~mm}$, and those measuring $\leq 2 \mathrm{~mm}$, the latter being called micrometastases. The extensive pathology examination of SLN has resulted in a frequent observation of micrometastases.

In the TNM classification of breast carcinomas, the micrometastases at SLN represent a subset of the nodepositive group (pN1mi) (9). We found micrometastasis in 96 of the SLN and we failed to observe differences in the overall survival and axillary recurrence, independent of micrometastasis size. We also know that our sample must be extended in the future to account for further considerations and to answer questions that remain unresolved. Specifically, what is the clinical significance of micrometastases? Also, should we perform axillary dissection or just attentive follow-up when micrometastases are found?

We observed a low rate of axillary metastasis (under $0.5 \%$ ) during follow-up of patients with clinically negative nodes that were not dissected at surgery, consistent with other studies (20). The lower incidences of axillary failure were likely due to systemic therapy and/or radiotherapy of

\section{References}

1. Giuliano AE, Kirgan DM, Guenther JM, Morton DL. Lymphatic mapping and sentinel lymphadenectomy for breast cancer. Ann Surg 1994; 220: 391-398.

2. Morton DL, Wen DR, Wong JH, Economou JS, Cagle LA, Storm FK, et al. Technical details of intraoperative lymphatic mapping for early stage melanoma. Arch Surg 1992; 127: 392-399.

3. van der Veen $\mathrm{H}$, Hoekstra OS, Paul MA, Cuesta MA, Meijer S. Gamma probe-guided sentinel node biopsy to select patients with melanoma for lymphadenectomy. Br J Surg 1994; 81: 1769-1770.

4. Veronesi U, Paganelli G, Viale G, Luini A, Zurrida S, Galimberti $V$, et al. A randomized comparison of sentinelnode biopsy with routine axillary dissection in breast cancer. N Engl J Med 2003; 349: 546-553.

5. Mansel RE, Fallowfield L, Kissin M, Goyal A, Newcombe RG, Dixon JM, et al. Randomized multicenter trial of sentinel node biopsy versus standard axillary treatment in operable breast cancer: the ALMANAC Trial. J Natl Cancer Inst 2006; the breast, which includes the lower axilla where most SLN were found. We note that 3 of the 4 cases that relapsed underwent intraoperative radiotherapy. Locoregional therapy has been demonstrated to improve not only disease-free survival, but also overall survival rates $(21,22)$. A short-term follow-up study suggested that the rate of regional recurrence may be very low $(0.12 \%)$ for patients considered to be SLN-negative on the basis of $\mathrm{H} \& \mathrm{E}$ and IHC sections and for those who do not undergo axillary dissection (14). In contrast, we note that $38.2 \%$ of SLN that test positive for macrometastasis contain another positive lymph node that is identified following paraffin analysis. The literature shows a high incidence of non-sentinel node involvement in cases of sentinel node macrometastasis, i.e., 39 to $79 \%$ of all cases, most of which depend on tumor size (23).

DCIS of the breast, by definition, cannot metastasize, unless there is an invasive component. In our study, SLNB in $\mathrm{DCIS}$ revealed isolated tumor cells $\leq 0.2 \mathrm{~mm}$ in two cases $(1.2 \%)$ and that the SLN was the only metastatic node found in the axillary dissection product. This is consistent with other literature reports (24). Thus, the real value of axillary dissection in DCIS is still questionable, especially when micrometastases or isolated tumor cells $\leq 0.2 \mathrm{~mm}$ are present. Intra et al. (25) reported a series of 854 patients with DCIS, of whom $1.4 \%$ had SLN metastasis. The ASCO guidelines recommend SLNB for patients with large or highgrade DCIS. The SLNB is still recommended for patients who subsequently undergo mastectomy for treatment of DCIS, because it becomes impossible to perform SLNB after removal of the breast (6).

In the future, we expect to attempt the use of SLNB for patients treated with neoadjuvant chemotherapy. In so doing, we plan to follow the consensus guidelines to improve the quality of life of early breast cancer patients.
98: 599-609.

6. Lyman $\mathrm{GH}$, Giuliano AE, Somerfield MR, Benson AB III, Bodurka DC, Burstein HJ, et al. American Society of Clinical Oncology guideline recommendations for sentinel lymph node biopsy in early-stage breast cancer. J Clin Oncol 2005; 23: 7703-7720.

7. Giuliano AE, Dale PS, Turner RR, Morton DL, Evans SW, Krasne DL. Improved axillary staging of breast cancer with sentinel lymphadenectomy. Ann Surg 1995; 222: 394-399.

8. Turner RR, Ollila DW, Krasne DL, Giuliano AE. Histopathologic validation of the sentinel lymph node hypothesis for breast carcinoma. Ann Surg 1997; 226: 271-276.

9. Sobin LH, Wittekind Ch. TNM classification of malignant tumors. 6th edn. New York: Wiley-Liss; 2007.

10. Singletary SE, Allred C, Ashley P, Bassett LW, Berry D, Bland KI, et al. Revision of the American Joint Committee on Cancer staging system for breast cancer. J Clin Oncol 2002; 20: 3628-3636.

11. Van Zee KJ, Manasseh DM, Bevilacqua JL, Boolbol SK, Fey 
JV, Tan LK, et al. A nomogram for predicting the likelihood of additional nodal metastases in breast cancer patients with a positive sentinel node biopsy. Ann Surg Oncol 2003; 10: 1140-1151.

12. Bevilacqua JL, Kattan MW, Fey JV, Cody HS III, Borgen PI, Van Zee KJ. Doctor, what are my chances of having a positive sentinel node? A validated nomogram for risk estimation. J Clin Oncol 2007; 25: 3670-3679.

13. Green FL, Page DL, Fleming ID, Fritz A, Balch CM, Haller DG, et al. AJCC cancer staging manual. 6th edn. New York: Springer; 2002.

14. Naik AM, Fey J, Gemignani M, Heerdt A, Montgomery L, Petrek $\mathrm{J}$, et al. The risk of axillary relapse after sentinel lymph node biopsy for breast cancer is comparable with that of axillary lymph node dissection: a follow-up study of 4008 procedures. Ann Surg 2004; 240: 462-468.

15. Veronesi U, Paganelli G, Galimberti V, Viale G, Zurrida $S$, Bedoni M, et al. Sentinel-node biopsy to avoid axillary dissection in breast cancer with clinically negative lymphnodes. Lancet 1997; 349: 1864-1867.

16. Takei H, Suemasu K, Kurosumi M, Horii Y, Yoshida T, Ninomiya J, et al. Recurrence after sentinel lymph node biopsy with or without axillary lymph node dissection in patients with breast cancer. Breast Cancer 2007; 14: 16-24.

17. Yi M, Meric-Bernstam F, Ross MI, Akins JS, Hwang RF, Lucci $A$, et al. How many sentinel lymph nodes are enough during sentinel lymph node dissection for breast cancer? Cancer 2008; 113: 30-37.

18. Motomura K, Egawa C, Komoike Y, Kataoka T, Nagumo S, Koyama $\mathrm{H}$, et al. Sentinel node biopsy for breast cancer: technical aspects and controversies. Breast Cancer 2007;
14: 25-30.

19. Clarke D, Leung E, Chachlani N, Rowlands D, Simon J, Hero I, et al. Intraoperative assessment of sentinel node using imprint cytology. World J Surg 2010; 34: 55-61.

20. Takei H, Kurosumi M, Yoshida T, Ninomiya J, Hagiwara $Y$, Kamimura M, et al. Current trends of sentinel lymph node biopsy for breast cancer - a surgeon's perspective. Breast Cancer 2007; 14: 362-370.

21. Ragaz J, Jackson SM, Le N, Plenderleith IH, Spinelli JJ, Basco VE, et al. Adjuvant radiotherapy and chemotherapy in node-positive premenopausal women with breast cancer. N Engl J Med 1997; 337: 956-962.

22. Overgaard M, Jensen MB, Overgaard J, Hansen PS, Rose $\mathrm{C}$, Andersson $\mathrm{M}$, et al. Postoperative radiotherapy in highrisk postmenopausal breast-cancer patients given adjuvant tamoxifen: Danish Breast Cancer Cooperative Group DBCG 82c randomised trial. Lancet 1999; 353: 1641-1648.

23. Nos C, Harding-MacKean C, Freneaux P, Trie A, Falcou MC, Sastre-Garau X, et al. Prediction of tumour involvement in remaining axillary lymph nodes when the sentinel node in a woman with breast cancer contains metastases. Br J Surg 2003; 90: 1354-1360.

24. Mabry H, Giuliano AE, Silverstein MJ. What is the value of axillary dissection or sentinel node biopsy in patients with ductal carcinoma in situ? Am J Surg 2006; 192: 455-457.

25. Intra $M$, Rotmensz $N$, Veronesi $P$, Colleoni $M$, lodice $S$, Paganelli G, et al. Sentinel node biopsy is not a standard procedure in ductal carcinoma in situ of the breast: the experience of the European Institute of Oncology on 854 patients in 10 years. Ann Surg 2008; 247: 315-319. 